\title{
HEREDITARY RADICALS AND BANDS OF ASSOCIATIVE RINGS
}

\author{
A. V. KELAREV \\ (Received 28 June 1989; revised 7 February 1990)
}

Communicated by B. J. Gardner

\begin{abstract}
Bands of associative rings were introduced in 1973 by Weissglass. For the radicals playing the most essential roles in the structure theory (in particular, for those of Jacobson, Baer, Levitsky, Koethe) it is shown how to find the radical of a band of rings. The technique of the general Kurosh-Amitsur radical theory is used to consider many radicals simultaneously.
\end{abstract}

1980 Mathematics subject classification (Amer. Math. Soc.) (1985 Revision): 16 A 21, 20 M 25.

Let $B$ be a band, that is, a semigroup of idempotents. An associative ring $R$ is called a band sum of the rings $R_{b}, b \in B$, or a $B$-sum of the rings $R_{b}$, if

(1) the additive group $R^{+}$is a direct sum of the $R_{b}^{+}$, and

(2) $R_{a} R_{b} \subseteq R_{a b}$ for any $a, b$ in $B$.

If $B$ is a semilattice, that is, a commutative band, then $R$ is called a semilattice sum of the rings $R_{b}, b \in B$. The rings $R_{b}$ are called the components of the band sum. The fact that $R$ is a $B$-sum of the rings $R_{b}$ will be denoted by $R=\bigoplus_{B} R_{b}$.

Band sums of rings were defined in [9]. The role of this concept has been discussed, for example, in [2] and [8]. Here we only note that band sums turn out to be useful when studying semigroup rings. For instance, in [6] a complete description of the Jacobson radical of semigroup algebras of commutative semigroups was obtained with the use of semilattice sums.

Let $\rho$ be a radical in the sense of Kurosh and Amitsur and $R=\bigoplus_{B} R_{b}$. The following problem seems to be natural: find the radical $\rho(R)$ provided

(C) 1991 Australian Mathematical Society 0263-6115/91 \$A2.00+0.00 
$\rho\left(R_{b}\right)$ are known. Some versions of this problem have been considered in the literature. For example, in [9] and [2] sufficient conditions for a semilattice sum of semisimple rings to be semisimple were considered, and in [2] the radicals $\rho$ were described such that if every component $R_{b}$ is radical, then $R$ is radical. In [4], [2], [5] and [6] the radicals $\rho$ commuting with a band $B$ were investigated. Recall that a radical $\rho$ is said to commute with $B$ sums if for every ring $R=\bigoplus_{B} R_{b}$ the equality $\rho\left(\bigoplus_{B} R_{b}\right)=\bigoplus_{B} \rho\left(R_{b}\right)$ holds. It follows from the results of [4] that the generalized nilradical of Andrunakievich and Rjabuhin commutes with every semilattice sum, and the radicals of Jacobson, Baer, Levitzky, Koethe and Brown-McCoy commute only with band sums having exactly one component.

The main result of the present paper (Theorem 1, see Section 1) solves the above posed problem for a rather wide class of radicals, including many important ones. The proof of Theorem 1 is contained in Section 2. In Section 3 we give an example showing that Theorem 1 is, in a sense, not improvable. In Section 4 we discuss some consequences of the main theorem and the results of [2].

The author is grateful to Professor L. N. Shevrin for his supervision, and to Dr. M. V. Volkov for useful discussions.

\section{Maín results}

We use standard definitions of the theory of radicals [1] and the theory of semigroups [3]. All the radicals playing essential roles in structure theory are hereditary. In this paper the word "radical" will mean "hereditary radical."

Let $\rho$ be a radical, $B$ be a band, and $R=\bigoplus_{B} R_{b}$. Recall that a semigroup is called a rectangular band if it satisfies the identity $x y x=x$. It is known $[3, \S 4.2$, Exercise 1] that there exist a semilattice $S$ and a homomorphism $f$ of $B$ onto $S$ such that every counterimage $Q_{s}=f^{-1}(s)$ is a rectangular band. In such a situation one says that $B$ is a semilattice $S$ of rectangular bands $Q_{s}$. Each $r$ in $R$ is uniquely represented as $r=\sum_{b \in B} r_{b}$, where $r_{b} \in R_{b}$ and all but a finite number of the $r_{b}$ are equal to zero. Consider on $S$ the natural order defined by $s_{1} \leq s_{2}$ if and only if $s_{1} s_{2}=s_{1}$. For $s \in B$ denote by $s$ the set of all $t \in B$ such that $f(t) \geq f(s)$. Set $r^{s}=\sum_{t \in s} r_{t}$. For any subset $A$ in $R$ set $A^{s}=\left\{a^{s} \mid a \in A\right\}$. Let $I(R, B, \rho)$ denote the set of all ideals $A$ in $R$ such that $A^{b} \cap R_{b} \subseteq \rho\left(R_{b}\right)$ for every $b \in B$. Let $\rho(R, B)$ be the sum of all ideals in $I(R, B, \rho)$. We shall say that $\rho$ is determined by the components of $B$-sums if and only if for every $R=\bigoplus_{B} R_{b}$ the equality $\rho(R)=\rho(R, B)$ holds. 
In Section 2 we shall show that if $\rho$ is determined by the components of $B$-sums then $\rho(R, B)$ is the largest ideal in $I(R, B, \rho)$, and therefore $\rho(R)$ may be described in terms of the $R_{b}$.

Now we consider some natural conditions on a radical. It is known that every radical class is closed under unions of ascending chains of ideals. A radical will be called $C$-local $\left(C_{r}\right.$-local, $C_{l}$-local $)$ if its radical class is closed under unions of ascending chains of subrings (right ideals, left ideals). For each radical $\rho$ the sum of two radical ideals in an arbitrary ring is radical. A radical $\rho$ will be called right summing (left summing) if and only if in every ring the sum of any two $\rho$-radical right ideals (left ideals) is $\rho$-radical. We call a radical $\rho$ right hereditary (left hereditary) if its radical class is closed under right ideals (left ideals). Recall that a radical is called supernilpotent if its radical class contains all nilpotent rings.

To consider simultaneously the interaction of band sums and many radicals let us introduce the following notion. A radical $\rho$ will be called countably definable if

(a) $\rho$ is $C$-local or there exists a nonradical ring $A$ being a union of an ascending chain of radical subrings $A_{1} \subseteq A_{2} \subseteq \cdots \subseteq A_{n} \subseteq \cdots$, where $n$ runs over the set of positive integers.

(b) $\rho$ is $C_{r}$-local $\left(C_{l}\right.$-local) or there exists a nonradical ring $A$ being a union of an ascending chain of radical right (left) ideals $A_{1} \subseteq A_{2} \subseteq \cdots \subseteq$ $A_{n} \subseteq \cdots$, where $n$ runs over the set of positive integers.

A considerable number of radicals known in the structure theory are countably definable. For example, the radicals of Jacobson, Levitzky, Baer, Koethe and Brown-McCoy belong to this class.

Recall that a semigroup is called a left (right) zero band if it satisfies the identity $x y=x \quad(x y=y)$. It is known and easy to prove that every rectangular band is a direct product of left zero band and right zero band.

Now we can state our main result.

THEOREM 1. Let $B$ be a band which is a semilattice $S$ of rectangular bands $Q_{s}$, where $Q_{s}$ is the direct product of a left zero band $L_{s}$ and a right zero band $P_{s}$. A countably definable radical $\rho$ is determined by the components of $B$-sums if and only if the following conditions hold:

(1) $S$ satisfies the descending chain condition or $\rho$ is $C$-local;

(2) every $L_{s}\left(P_{s}\right)$ is finite or $\rho$ is $C_{r}$-local $\left(C_{l}\right.$-local);

(3) every $L_{s}\left(P_{s}\right)$ consists of one element or is right (left) hereditary, right (left) summing and supernilpotent.

Now we apply the theorem to some radicals frequently used in the structure theory. 
COROLlaRY 1. The Jacobson and the Levitzky radicals are determined by the components of every band sum.

Corollary 2. Let $\rho$ be the Brown-McCoy or Andrunakievich-Rjabuhin radical. Then $\rho$ is determined by the components of $B$-sums if and only if $B$ is a semilattice.

COROllary 3. Let $B$ be a band which is a semilattice $S$ of rectangular bands. The Baer radical is determined by the components of B-sums if and only if $S$ satisfies the descending chain condition.

COROLlary 4. If the Koethe problem has positive solution then the Koethe radical is determined by the components of every band sum. If the Koethe problem has negative solution then the Koethe radical is determined by the components of $B$-sums if and only if $B$ is a semilattice.

\section{Proof of the main theorem}

The proof of [2, Lemma 1.2] gives us

Lemma 1 . Let $S$ be a semilattice, $\rho$ a radical, and $R=\bigoplus_{S} R_{s}$. Then $\rho(R) \in I(R, S, \rho)$.

LEMMA 2. Let $P$ be a right (left) zero band, $\rho$ be left (right) hereditary radical, and $R=\bigoplus_{P} R_{p}$. Then $\rho(R) \in I(R, P, \rho)$.

Proof. For any $p \in P$ the intersection $\rho(R)^{b} \cap R_{b}$ is a right ideal in $R$, since $R_{b}$ is right ideal in $R$. By the right hereditariness, $\rho(R)^{b} \cap R_{b} \subseteq \rho\left(R_{b}\right)$. It means that $\rho(R) \in I(R, P, \rho)$, which completes the proof.

We shall use the following notation. Let $R=\bigoplus_{B} R_{b}, b \in B, P \subseteq B$, $A \subseteq R$. Then we set $A_{b}=A \cap R_{b}, A_{P}=A \cap \sum_{p \in P} R_{p}$.

Proof of Theorem 1. Denote by $\mathscr{R}$ the radical class of $\rho$.

(a) Necessity. Let $\rho$ be determined by the components of $B$-sums. We are to prove that conditions (1) to (3) hold.

First we suppose that condition (1) does not hold, that is, $S$ contains a descending chain $s_{1}>s_{2}>\cdots$ and $\rho$ is not $C$-local.

Since $\rho$ is countably definable there exists a nonradical ring $A$ which is a union of radical subrings $A_{1} \subseteq A_{2} \subseteq \cdots \subseteq A_{n} \subseteq \cdots$, where $n$ runs over the set of positive integers. Choose elements $q_{i}$ in $Q_{s_{i}}, i=1,2, \ldots$, and 
set $t_{1}=q_{1}, t_{i+1}=t_{i} q_{i} t_{i}$. Then the set $T=\left\{t_{1}, t_{2}, \ldots\right\}$ is a subsemilattice in $B$. For $t \in T, t=t_{i}$, denote by $R_{t}$ the subring $A_{i} t_{i}$ of the semigroup ring $A T$. For $b \in B \backslash T$ set $R_{b}=0$. Let $R=\sum_{b \in B} R_{b}$. Clearly $R$ is a $B$-sum of the $R_{b}$. Each ring $R_{b}$ is isomorphic to some $A_{i}$ or is equal to zero. Thus all $R_{b}$ are radical. Since $\rho$ is determined by the components of $B$-sums, we have $R \in \mathscr{R}$. Denote by $\varphi$ the homomorphism of $A T$ onto $A$, defined by the rule $\varphi\left(\sum_{i=1}^{n} a_{i} b_{i}\right)=\sum_{i=1}^{n} a_{i}$. Recall that $R$ is contained in $A T$. Clearly $\varphi$ maps $R_{t_{i}}$ on $A_{i}$. Hence $\varphi(R)=A$. Since every radical class is closed under homomorphisms, $A \in \mathscr{R}$. This contradicts the choice of $A$.

Now we shall deal with conditions (2) and (3). These conditions contain two dual assertions, and in both cases we shall consider only one assertion.

Suppose that condition (2) does not hold, that is, a certain band $L_{s}$ is infinite and $\rho$ is not $C_{r}$-local.

Since $\rho$ is countably definable, there exists a nonradical ring $A$ which is a union of radical right ideals $A_{1} \subseteq \cdots \subseteq A_{n} \subseteq \cdots$, where $n$ runs over the set of positive integers. Obviously $B$ contains a subsemigroup isomorphic to $L_{s}$. We identify this semigroup with $L_{s}$, so that $L_{s} \subseteq B$. Choose in $L_{s}$ a countable subset $L$ and index the elements of $L$ by the positive integers $L=\left\{b_{1}, b_{2}, \ldots\right\}$. Denote by $R_{b_{i}}$ the subring $A_{i} b_{i}$ of the semigroup ring $A L$. For $b \in B \backslash L$ set $R_{b}=0$. Let $R=\sum_{b \in B} R_{b}$. Clearly $R$ is a $B$-sum of the $R_{b}$, and all $R_{b}$ belong to $\mathscr{R}$. Therefore $\rho(R)=\rho(R, B)=R$. Denote by $\varphi$ the homomorphism of $A L$ in $A$ defined by the rule $\varphi\left(\sum_{i=1}^{n} a_{i} b_{i}\right)=$ $\sum_{i=1}^{n} a_{i}$. Recall that $R$ is contained in $A L$. Evidently $\varphi$ maps $R$ onto $A$. Hence $A \in \mathscr{R}$, giving a contradiction.

Now we shall prove that condition (3) holds. Clearly it suffices to consider the case where $L_{s}$ is not a singleton, and so $L_{s}$ contains a two-element band $L=\{c, d\}$. Identifying $L$ with a subband of $B$, we may assume that $L \subseteq B$.

We claim that $\rho$ is right summing. Take any ring $A$ which is a sum of radical right ideals $I$ and $J$. Consider in the semigroup ring $A L$ subrings $R_{c}=I c$ and $R_{d}=J d$. For $b \in B \backslash L$ set $R_{b}=0$. Then the ring $R=R_{c}+R_{d}$ is a $B$-sum of the radical rings $R_{b}$. Hence $R \in \mathscr{R}$. Denote by $\varphi$ the homomorphism of $A L$ in $A$ defined by the rule $\varphi\left(a_{1} c+a_{2} d\right)=a_{1}+a_{2}$. Clearly $\varphi$ maps $R$ onto $A$, hence $A$ is radical. We have proved that $\rho$ is right summing.

Now we prove that $\rho$ is supernilpotent. Take any ring $A$ with zero multiplication. Consider the semigroup ring $A L$ and set $R_{b}=A b$ when $b \in L$, $R_{b}=0$ when $b \in B \backslash L$. Then $A L$ is a $B$-sum of the $R_{b}$. Denote by $C$ the ideal of $A L$ consisting of all elements of the form $a c-a d$, where $a \in A$. Obviously $C \in I(A L, B, \rho)$, implying $C \subseteq \rho(A L)$ and $C \in \mathscr{R}$. Clearly 
$A \cong C$. Thus $A \in \mathscr{R}$. We have proved that $\rho$ is supernilpotent.

We claim that $\rho$ is right hereditary. Take any radical ring $A$ and its right ideal $I$. In the semigroup ring $A L$ set

$$
R_{b}= \begin{cases}A b & \text { when } b \in L, \\ 0 & \text { when } b \in B \backslash L .\end{cases}
$$

Then the ring $A L=R_{c}+R_{d}$ is a $B$-sum of the $R_{b}$. Since $\rho$ is determined by the components of $B$-sums, we have $A L \in \mathscr{R}$. Denote by $J$ the ideal of $A L$ consisting of all elements $i c-i d$, where $i \in I$. By the hereditariness of $\rho$ the ideal $J$ is radical. Set $R=A c+I d$. The quotient ring $R / J$ is radical, because it is isomorphic to $A$. Since $\mathscr{R}$ is closed under extensions, we have $R \in \mathscr{R}$. Further $R$ is a $B$-sum of the rings $Q_{b}$, where

$$
Q_{b}= \begin{cases}A C & \text { when } b=c, \\ I d & \text { when } b=d, \\ 0 & \text { when } b \in B \backslash L .\end{cases}
$$

Thus $\rho(R, B)=R$. For every $T \in I(R, B, \rho)$ we get $I d T \subseteq T \cap I d=T^{d} \cap$ $I d \subseteq \rho(I d)$. Therefore $I d R=I d \rho(R, B) \subseteq \rho(I d)$, and so $(I d)^{2} \subseteq \rho(I d)$. Since $\rho$ is supernilpotent, $I d \in \mathscr{R}$. Clearly $I \cong I d$ and so $I$ is radical. We have proved that $\rho$ is right hereditary.

Thus $\rho$ is right summing, right hereditary and supernilpotent. This means that condition (3) holds. This completes the proof of necessity.

(b) Sufficiency. The proof of sufficiency will be made in five steps. Four of them will deal with the partial cases where $B$ is a semilattice (that is, $B=S$ ), and $B$ is a left zero band (that is, $B=L_{s}$ ), and $B$ is a right zero band (that is, $B=P_{s}$ ), and $B$ is a rectangular band (that is, $B=Q_{s}$ ). The fifth step will complete the proof of the general case.

STEP 1. Let $B$ be a semilattice, that is, $B=S$. Take any $R=\bigoplus_{S} R_{s}$. We claim that $\rho(R)$ is the largest ideal in $I(R, S, \rho)$.

By Lemma $1, \rho(R) \in I(R, S, \rho)$. On the other hand, for any $I$ in $I(R, S, \rho)$ we are to prove that $I \subseteq \rho(R)$. In view of condition (1) there are two possibilities.

CASE 1. $S$ satisfies the descending chain condition.

By induction we shall define ideals $S_{1}, S_{2}, \ldots$ of $S$. Let $S_{1}=\left\{s_{1}\right\}$, where $s_{1}$ is the least element in $S$. Suppose that for an ordinal number $\nu$ the ideals $S_{\mu}, \mu<\nu$, are defined. If $\nu$ is a limit ordinal (that is, the number $\nu-1$ does not exist), then we set $S_{\nu}=\bigcup_{\mu<\nu} S_{\mu}$. Otherwise, we choose a minimal element $s_{\nu}$ in $S \backslash S_{\nu-1}$ and set $S_{\nu}=S_{\nu-1} \cup\left\{s_{\nu}\right\}$. Clearly, the process will terminate, and we shall have $S_{\tau}=S$. Let $Q_{\nu}=I \cap \sum_{\mu \leq \nu} R_{s_{\mu}}$ 
where $\nu \leq \tau$. An easy induction shows that $S_{\nu}$ is an ideal in $S$, and so $Q_{\nu}$ is an ideal in $I$. Evidently, $I=Q_{\tau}$.

Now we shall prove every $Q_{\nu}$ to be in $\mathscr{R}$. Since $I$ is in $I(R, S, \rho)$, we get $Q_{1}=I \cap R_{s_{1}} \subseteq \rho\left(R_{s_{1}}\right)$, and so $Q_{1} \in \mathscr{R}$. Suppose that for some $\nu$ the rings $Q_{\mu}, \mu<\nu$, are in $\mathscr{R}$. If $\nu$ is a limit ordinal, then $Q_{\nu}$ is a union of the ideals $Q_{\mu}, \mu<\nu$, implying $Q_{\nu} \in \mathscr{R}$. If $\nu$ is not a limit ordinal, then $Q_{\nu}$ is an extension of $Q_{\nu-1}$ by $\left(Q_{\nu}\right)^{s} \cap R_{s}$. The latter is an ideal in $I^{s} \cap R_{s} \subseteq \rho\left(R_{s}\right)$. Therefore $Q_{\nu}$ is radical too.

Since $I=Q_{\tau}$, we have $I \in \mathscr{R}$ as required.

CASE 2. $\rho$ is $C$-local.

First we introduce the following technical definition. Let $\mathscr{F}$ be the set of all finite subsemigroups of $S, \tau$ be an ordinal number, and suppose $S_{1}, \ldots, S_{\tau}$ are semigroups of $\mathscr{F}$ such that for any limit ordinal $\mu \leq \tau$ the semigroup generated in $S$ by the semigroups $S_{\nu}, \nu<\mu$, contains $S$. Let $T$ denote the semigroup generated in $S$ by $\bigcup_{\xi<\nu} S_{\xi}$. Set $I_{\nu}=I_{T_{\nu}}$. Then the ascending chain of rings $I_{1} \subseteq I_{2} \subseteq \cdots \subseteq I_{\tau} \subseteq \cdots$ will be called a $\tau$-chain in $I$.

Obviously $I$ is equal to the union of a $\tau$-chain in $I$ for suitable $\tau$. Thus for proving sufficiency it remains to show that the union of any $\tau$-chain in $I$ is radical. This will be done by transfinite induction.

Let $I_{1}$ be a 1 -chain generated by a finite semilattice $S_{1}$. Then $R_{S_{1}}$ is an $S_{1}$-sum of the $R_{s}$. Since $S_{1}$ satisfies the descending chain condition, case 1 yields us that $\rho$ is determined by the components of $S_{1}$, that is, $\rho\left(R_{S_{1}}\right)=\rho\left(R_{S_{1}}, S_{1}\right)$. Since $I_{1}=I_{S_{1}}$ and $I \in I(R, S, \rho)$ then $I_{1}$ is an ideal in $\rho\left(R_{S_{1}}, S_{1}\right)$. Hence $I_{1} \subseteq \rho\left(R_{S_{1}}\right)$, implying $I_{1}$ is radical. Suppose that for each $\nu<\tau$ the union of every $\nu$-chain in $I$ is proved to be radical. Consider any $\tau$-chain $I_{1} \subseteq I_{2} \subseteq \cdots \subseteq I_{\tau}$ generated by finite semigroups $S_{1}, S_{2}, \ldots, S_{\tau}$. If $\tau$ is a limit ordinal, then $I_{\tau}=\bigcup_{\nu<\tau} I_{\nu}$ and so $I_{\tau}$ is radical since $\rho$ is $C$-local. Further we consider the case where $\tau$ is not a limit ordinal. Set $P_{\nu}=T_{\nu} S_{\tau}, A_{\nu}=I_{P_{\nu}}$. Clearly $A_{\tau-1}$ is equal to the union of ( $\tau-1$ )-chain $A_{1} \subseteq A_{2} \subseteq \cdots \subseteq A_{\tau-1}$ generated by the finite semigroups $P_{1}, P_{2}, \ldots, P_{\tau-1}$. By the induction hypothesis $A_{\tau-1}$ is radical. Then the sum $J=I_{\tau-1}+A_{\tau-1}$ is radical. By the choice of $I$ the quotient ring $I_{\tau} / J$ is an ideal in $\rho\left(R_{S_{\tau}}, S_{\tau}\right) / J$. In view of Case $1, \rho\left(R_{S_{\tau}}, S_{\tau}\right)$ is radical. Therefore $I_{\tau} / J$ is radical, implying $I_{\tau} \in \mathscr{R}$.

Thus we have proved that $I$ is radical. Hence $\rho(R, S)$ is the largest ideal in $I(R, S, \rho)$.

STEP 2. Let $B$ be a left zero band, and $R$ be a $B$-sum of the rings $R_{b}$. We claim that $\rho(R)$ is the largest ideal in $I(R, B, \rho)$. 
By Lemma 2, $\rho(R)$ lies in $I(R, B, \rho)$. On the other hand let us take any ideal $I$ in $I(R, B, \rho)$ and prove that $I \subseteq \rho(R)$.

Set $A_{b}=R_{b} I$ and $A=\sum_{b \in B} A_{b}$. Clearly $A=R I$ and $A_{b}=A \cap R_{b}$. Then $A_{b} \subseteq I \cap R_{b} \subseteq \rho\left(R_{b}\right)$, and so $A_{b}$ is radical. If $B$ is finite then $A$ is a finite sum of radical right ideals $A_{b}$. Since $\rho$ is right summing, we have $A \in \mathscr{R}$. Now we shall consider the case where $B$ is infinite and prove that $A$ is in $\mathscr{R}$. For any subband $T$ in $B$ set $A_{T}=A \cap \sum_{b \in T} R_{b}$. Obviously $A_{T}$ is a right ideal in $R$. By condition (2), $\rho$ is $C_{r}$-local. By Zorn's Lemma there is a subsemigroup $T$ in $B$ maximal with the property $A_{T} \in \mathscr{R}$. If $T \neq B$, then for $b \in B \backslash T$ we have $Q_{T \cup\{b\}}=A_{T}+A_{b} \in \mathscr{R}$, contradicting the choice of $T$. Therefore $T=B$ and $A=A_{B} \in \mathscr{R}$. Further $I^{2}$ is an ideal in $A$, and hence $I^{2} \in \mathscr{R}$. By condition (3), $\rho$ is supernilpotent, implying $I / I^{2} \in \mathscr{R}$ and so $\rho(R) \supseteq I$. Thus $\rho(R)$ is the largest ideal in $I(R, B, \rho)$.

STEP 3. Let $B$ be a right zero band and $R=\bigoplus_{B} R_{b}$. Then dually to Step 2 we may prove that $\rho(R)$ is the largest ideal in $I(R, B, \rho)$.

STEP 4 . Let $B$ be a rectangular band and $R=\bigoplus_{B} R_{b}$. We claim that $\rho(R)$ is the largest ideal in $I(R, B, \rho)$.

For a suitable left zero band $L$ and a suitable right zero band $P$ we have $B \cong L \times P$. If $L$ or $P$ is one-element, then $B$ is isomorphic to $P$ or $L$ and Steps 2 and 3 complete the proof. Further we consider the case where both $L$ and $P$ have at least two elements.

Choose $q$ in $B$ and set $A=\sum_{b \in B_{q}} R_{b}$. Evidently, $A$ is a left ideal in $R$ and $R_{q}$ is a right ideal in $A$. By condition (3), $\rho$ is right and left hereditary. Hence $\rho(R) \cap R_{q}=(\rho(R) \cap A) \cap R_{q} \subseteq \rho(A) \cap R_{q} \subseteq \rho\left(R_{q}\right)$. We have proved that $\rho(R) \in I(R, B, \rho)$.

On the other hand take any $I$ in $I(R, B, \rho)$. We are to prove that $I \subseteq$ $\rho(R)$. For any $b$ in $L$ set $C_{b}=\sum_{p \in P} R_{(b, p)}$. Then $R=\bigoplus_{L} C_{b}, C_{b}=$ $\bigoplus_{P} R_{(b, p)}$. Obviously $I \cap C_{b} \in I\left(C_{b}, P, \rho\right)$. By Step 2 this implies $I \cap C_{b} \subseteq$ $\rho\left(C_{b}\right)$. In view of Step 3 it follows that $I \subseteq \rho(R)$ as required.

STEP 5. Let $B$ be an arbitrary band which is a semilattice $S$ of rectangular bands $Q_{s}$, where $Q_{s}$ is a direct product of a left zero band $L_{s}$ and a right zero band $P_{s}$. Let $R=\bigoplus_{B} R_{b}$. We claim that $\rho(R)$ is the largest ideal among the ideals of $I(R, B, \rho)$.

Take any $b$ in $B$. Let $b$ be in $Q_{s}, s \in S$. Set $Q=Q_{s}$. Lemma 1 yields $\rho(R)^{s} \cap R_{Q} \subseteq \rho\left(R_{Q}\right)$. By Step 4, $\rho\left(R_{Q}\right) \in I\left(R_{Q}, Q, \rho\right)$. Since $\rho(R)^{s}=\rho(R)^{b}$, we get $\rho(R)^{b} \cap R_{b} \subseteq \rho\left(R_{Q}\right) \cap R_{b} \subseteq \rho\left(R_{b}\right)$. This means that $\rho(R) \in I(R, B, \rho)$.

On the other hand take any $A$ in $I(R, B, \rho)$. For any $s$ in $S$ and $Q=Q_{s}$, the intersection $A^{s} \cap R_{Q}$ is in $I\left(R_{Q}, Q, \rho\right)$. Therefore, by Step 
4, $A^{s} \cap R_{Q} \subseteq \rho\left(R_{Q}\right)$. Hence $A \in I(R, S, \rho)$. By Step 1 we get $A \subseteq \rho(R)$. This completes the proof.

\section{An example of a radical which is not countably definable}

Theorem 1 says that a countably definable radical is determined by the components of a semilattice $S$ if and only if it is determined by the components of $C$-sums for every chain $C$ contained in $S$. A natural question arises: does the analogous statement hold for an arbitrary radical? Here we shall show that the answer is negative. Thereby an extension of Theorem 1 for arbitrary radicals does not hold.

Proposition 1. There are a radical $\rho$ and a semilattice $S$ such that $\rho$ is determined by the components of $C$-sums for every chain $C$ in $S$ but $\rho$ is not determined by the components of $S$-sums.

Proof. Let $F$ be a field. For an ordinal number $\mu$ the ring of $(\mu \times \mu)$ matrices over $F$ with finite supports will be denoted by $F_{\mu}$. Recall that a subring $A$ of the ring $R$ is called a metaideal in $R$ if and only if there is a finite series of rings $A=A_{0} \subseteq A_{1} \subseteq \cdots \subseteq A_{n}=R$ such that $A_{i-1}$ is an ideal in $A_{i}$ for $i=1, \ldots, n$. Let $\tau$ be the least uncountable ordinal number and let $\mathscr{R}$ be the class of rings $R$ such that every quotient ring $R / I$ has no subring isomorphic to $F_{\tau}$. It is routine to verify that $\mathscr{R}$ is closed under ring extensions, quotient rings and sums of ideals. Hence by [1, Theorem 2.2.1], $\mathscr{R}$ is a radical class. Let $\rho$ be the radical with the radical class $\mathscr{R}$.

Let $S$ be the set of all finite sets consisting of ordinal numbers which are less than $\tau$. Clearly $S$ is a semilattice with respect to the union of sets.

First we prove that $\rho$ is not determined by the components of $S$-sums. For $s \in S$, let $A_{s}$ denote the set of matrices $M$ in $F_{\tau}$ such that $\nu<\tau$, $\nu \notin s$ implies that the $\nu$-column and the $\nu$-row of $M$ contain only zeros. Obviously $A_{s}$ is radical. Consider the semigroup ring $F_{\tau} S$ and set $R_{s}=A_{s} s$, where $s \in S$. Let $R=\sum_{s \in S} R_{s}$. Then $R$ is an $S$-sum of the $R_{s}$. Since the $R_{s}$ are radical, we have $\rho(R, S)=R$. Consider the homomorphism $\varphi$ of $F_{\tau} S$ in $F_{\tau}$ defined by the rule $\varphi\left(\sum_{i=1}^{n} f_{i} s_{i}\right)=\sum_{i=1}^{n} f_{i}$. Then $\varphi(R)=F_{\tau}$. Evidently $F_{\tau}$ is semisimple, and so $R$ is not radical. Hence $\rho(R) \neq \rho(R, S)$. Thus $\rho$ is not determined by the components of $S$-sums.

Further, let $C$ be a chain lying in $S$. Then $C$ is countable. Therefore in proving that $\rho$ is determined by the components of $C$-sums it suffices to show that $\mathscr{R}$ is closed under countable ascending chains of subrings. Then 
the proof of Theorem 1 (namely, Case 2 of Step 1) may be applied to $\rho$ and $C$ without any changes.

Now we prove that $\mathscr{R}$ is closed under unions of countable ascending chains of rings. Suppose the contrary. Then there is a ring $R$ containing $F_{\tau}$ which is a union of a countable ascending chain of $\mathscr{R}$-rings $R_{i}, i \in I$. Denote by $e_{\mu, \nu}$, where $\mu, \nu<\tau$, the matrix in $F_{\tau}$ with the only nonzero element 1 at the intersection of the $\mu$-row and the $\nu$-column. For $\nu<\tau$ set $p_{\nu}=\left\{e_{\nu 1}, e_{1 \nu}\right\}$. Since the chain $A_{i}, i \in I$, is ascending, each pair is contained in some $A_{i}$. Let $M_{i}$ be the set of ordinal numbers $\nu$ such that $p_{\nu} \subseteq A_{i}$. The set of ordinal numbers which are less than $\tau$ is uncountable. Hence $M_{i}$ is uncountable for some $i$. Therefore $M_{i}$ has the same cardinality as $\tau$. Let $A$ denote the ring generated in $F_{\tau}$ by the $p_{\nu}$, where $\nu \in M_{i}$. For any $\mu, \nu$ in $M_{i}$ the ring $A$ contains the matrices $e_{\mu 1}, e_{1 \nu}$ and so contains also $e_{\mu \nu}$. Hence $A$ is isomorphic to $F_{\tau}$, contradicting $A \in \mathscr{R}$. Thus $\mathscr{R}$ is closed under unions of countable ascending chains of rings. Therefore $\rho$ is determined by the components of $C$-sums, which completes the proof.

\section{A connection of $C$-localness and other conditions}

Let $R$ be a ring, $R_{i}(i \in I)$ subrings of $R$, and put $M=\left\{R_{i}, i \in I\right\}$. The system $M$ is said to be a local system of $R$ if and only if for every $i, j$ in $I$ there is $k$ such that $R_{k} \supseteq R_{i}+R_{j}$, and $\bigcup_{i \in I} R_{i}=R$. Following [2], we say that $M$ is a directed system if for every $i, j$ in $I$ the set $\left\{R_{k} \mid R_{k} \supseteq R_{i}+R_{j}\right\}$ contains a minimal ring, and $\bigcup_{i \in I} R_{i}=R$. A radical class $\mathscr{R}$ is called local (directed) if and only if for every ring $R$ with a local (directed) system $\left\{R_{i}\right.$, $i \in I\}$ all $R_{i} \in \mathscr{R}$ implies $R \in \mathscr{R}$. Recall that $\mathscr{R}$ is said to be closed under semilattice sums if and only if for every semilattice $S$ and every ring $R=\bigoplus_{S} R_{s}$ all $R_{i} \in \mathscr{R}$ implies $R \in \mathscr{R}$. Now [2, Theorem 3.2] tells us that $\mathscr{R}$ is closed under semilattice sums if and only if it is directed. Obviously every directed class is $C$-local. Theorem 1 implies that every $C$-local radical class is closed under semilattice sums, and so it is directed. Thus we may state

Proposition 2. A radical class is directed if and only if it is C-local.

The definitions of local and directed radicals are very close. However, the following question is still open.

QUESTION. Is there a directed radical which is not local? 


\section{References}

[1] V. A. Andrunakievich and Ju. M. Rjabuhin, Radicals of algebras and structure theory (Nauka, Moscow, 1979), in Russian.

[2] H. L. Chick and B. J. Gardner, 'The preservation of some ring properties by semilattice sums', Comm. Algebra 15 (1987), 1667-1677.

[3] A. Clifford and G. Preston, The algebraic theory of semigroups, (Math. Surveys No. 7 Amer. Math. Soc., Providence, R.I., 1964).

[4] B. J. Gardner, 'Radicals and supplementary semilattice sums of associative rings', Pacific J. Math. 58 (1975), 387-392.

[5] A. V. Kelarev, 'Radicals and bands of associative algebras', Algebra $i$ logica (Novosibirsk) 28 (1989), 430-443.

[6] V. A. Kelarev, 'Radicals of semigroup algebras of commutative semigroups', Abstracts of XIX All-Union algebraic conference (Lvov, 1987), Vol. 2, 122, in Russian.

[7] A. V. Kelarev, 'Radicals of semilattices of algebras and semigroup algebras', Vestnik Moscov. Gos. Univ. No. 173 (1988), in Russian.

[8] M. V. Volkov and A. V. Kelarev, 'Varieties and bands of associative rings', Izv. Vyssh. Ucebn. Zaved. Mat. No. 9, (1986), 16-23, in Russian.

[9] J. Weissglass, 'Semigroup rings and semilattice sums of rings', Proc. Amer. Math. Sac. 39 (1973), 471-478.

\section{Department of Mathematics \\ Ural State University \\ Lenina 51 \\ Sverdlovsk \\ USSR 620083}

